\title{
Combining the radiative, conductive and convective heat flows in and around a skylight
}

\author{
Martin Fält", Ron Zevenhoven \\ Thermal and Flow Engineering, Department of Chemical Engineering, Åbo Akademi University, \\ Turku, 20500, Finland \\ *Corresponding author. Tel: +358 2215 4441, Fax: +358 2215 4792, E-mail: martin.falt@abo.fi
}

\begin{abstract}
Normal skylights bring light into the spaces located below them. By the use of infrared radiation (IR) transmissive polymer films and IR-emitting and absorbing gases, an advanced version of the skylight may supply cooling and thermal insulation to the room located below it. This novel radiative skylight can, in its cooling mode, lead heat from the room below, to the cool skies located above the skylight. When cooling is no longer needed or attainable this connection will be cut, thus providing the room with an optimal amount of thermal resistance. This article is a progress report on the modeling of the skylight. The main work is done to combine the different heat transfer methods into one single model by the use of the commercial program Comsol 4.1. The results show that a cooling effect of $100 \mathrm{~W} / \mathrm{m}^{2}$ is achievable when the skylight is compared to a similar skylight containing only air.
\end{abstract}

Keywords: Radiative cooling, heat transfer in participating media, skylight.

\section{Introduction}

Skylights are popular in building technology due to their ability to bring light into a space. However, as they light up a space, they also heat it up. This heat can be unwanted and therefore, has to be removed. This article will present the results from a numerical modeling of a skylight, which can function either as a radiative cooler or as a thermal insulator. Radiative cooling is a passive cooling method that connects a warm object located e.g. on top of a building to a lower sky temperature through heat radiation; one could describe it as an inverse solar collector [1].

The improved skylight contains a quantity of gas that is active in radiative heat transfer (i.e. a participating gas), and a cooling or insulating effect is achieved by controlling the circulating motion of this gas. The use of gases in the spaces between windows has been studied mainly with the goal of increasing thermal resistance by replacing air with a gas having lower conductive properties. Another option is to use gases that absorb and emit thermal radiation and thus decrease the radiative heat transfer through the window [2]. The use of such gases, which are active in radiative heat transfer, has also been studied for radiative cooling purposes; these studies show that cooling is also attainable during the day [3-5]. However, a window or a skylight that combines the three functions is a novel idea.

\section{Methodology}

The skylight model, whose design and function is described in [6], was designed at the authors' laboratory at Åbo Akademi University to determine its performance as a passive cooler and a thermal insulator. As shown in Figure 1, it consists of three windows. The outer and the inner window are made of an IR-transmitting polymer, $\tau=0.19$, and the middle window is made of silica glass, which is highly reflective to IR radiation at all wavelengths, $\rho=0.9$. The spaces between these windows contain a greenhouse gas that acts as the system's heat carrier. The walls are assumed to be thermally insulated and to have an absorptivity of $\alpha=0.9$. Heat that originates directly from the sun is not incorporated into this model as both 
the windows and the gas are assumed not to absorb short wave heat radiation in the interval of $0.1-3 \mu \mathrm{m}$.

When the skylight is set in its cooling mode, heat is transferred to the gas through the lower window (Glass 1) by radiation, conduction, and convection from the room below. The heated gas rises to the upper compartment due to the decrease in the gases' density. In the upper space, the gas is cooled by radiative heat transfer through the upper polymer window (Glass 2) and the "atmospheric window" to the colder air masses in the upper atmosphere. There also exists a forced convective heat transfer between the upper part of the skylight and its surroundings. The effect of this heat transfer depends on the temperature of the surroundings; for the modeled cases, it is cooling.

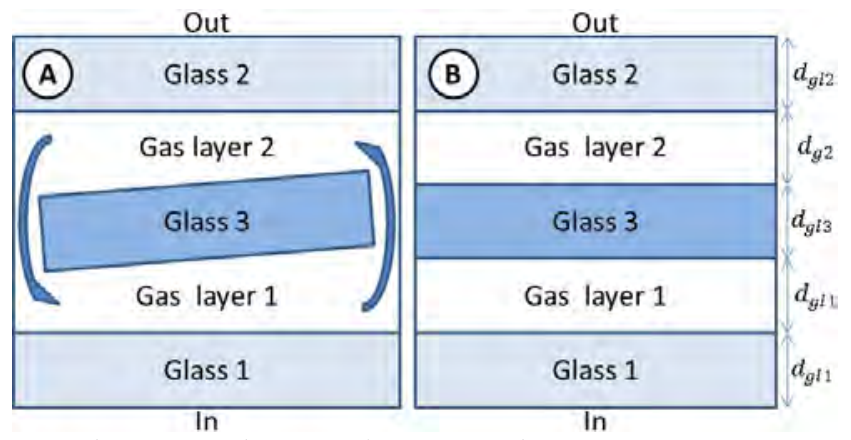

Fig. 1.Skylight in cooling mode "A" and in insulating mode "B"

When the greenhouse gas cools, its density increases, and it flows down to the lower part of the skylight. This convective heat flow is induced by the slightly tilted middle window. The angel of the tilt and the width of glass 3 has been chosen according to Fig. 2 so as to decrease the formation of hindering Bérnard cells (convective swirls).

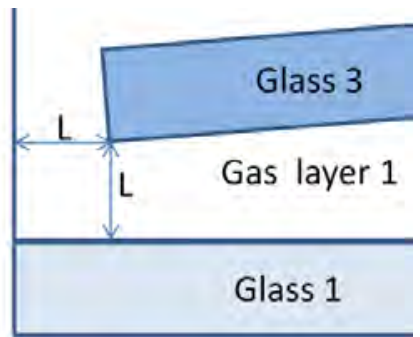

Fig. 2. Detail of the skylight in cooling mode

Then when no cooling is needed, the connection between the two gas spaces is cut, thus changing the task of the roof components from a passive radiative cooler to a thermal insulator. The weather parameters that are used in this modeling are average values for the months of February and July for year 2008 in Helsinki, Finland. The data for February is used to model when a skylight with a maximal amount of thermal resistance is needed and the data for July is used to model when cooling is needed. The data was procured from the Finnish meteorological institute, and it is presented in Table 1.

Table 1. Average weather data for two months in 2008 for Helsinki Finland.

\begin{tabular}{ccc}
\hline Unit & February & July \\
\hline $\mathrm{T}_{\text {ambient }}\left[{ }^{\circ} \mathrm{C}\right]$ & 0.73 & 17.62 \\
$\mathrm{~T}_{\text {sky }}\left[{ }^{\circ} \mathrm{C}\right]$ & -7.51 & 3.67 \\
$\mathrm{~V}_{\text {wind }}[\mathrm{m} / \mathrm{s}]$ & 4.13 & 3.20 \\
\hline
\end{tabular}


Modeling natural convection induced by heat radiation is, however, somewhat tricky; an attempt to do this was made by the author in [6]. It has also been shown that the wavelength dependency is crucial for calculating radiative heat transfer in participating media [7].

The exponential wide-band method is used in this paper to calculate the gases absorptivity and emissivity, which are assumed to be equal [8]. The studied gas is chosen to be $\mathrm{CO}_{2}$ which absorptivity is calculated to be, $\alpha=0.19$, as an isothermal and a gray value (no wave length dependency). In future work the gas absorptivity will be treated as temperature and wavelength dependent variable.

\section{Results}

The results from the modeling are given in Table 2 where the total heat transfers from the room to the skylight are presented. This data is calculated by the Comsol model in $\mathrm{W} / \mathrm{m}$ as the model is 2 dimensional. These results are in turn squared to get them into a more comfortable unit of $\mathrm{W} / \mathrm{m}^{2}$. These results are from the last time step of a 1000 second long dynamic simulation. The reason for solving this problem as a time dependent instead of a stationary problem is to avoid unstable equilibrium points. The time of 1000 seconds was assumed to be a time period long enough for the heat flows to stabilize. However, some of the simulations seem not to have stabilized.

Table 2 shows that a cooling capacity $98 \mathrm{~W} / \mathrm{m}^{2}$ can be obtained during the summer by this design, when comparing the cooling case with $\mathrm{CO}_{2}$ to the insulating case with air. The table also shows that if the skylight is set in its insulating mode for the winter an unnecessary heat loss of $80 \mathrm{~W} / \mathrm{m}^{2}$ would be achieved. This same effect is also achieved if the skylight is filled with air. A reason to this could be that the simulations were not simulated for a long enough time.

Table 2. Average heat transfer for two months in 2008 for Helsinki, Finland.

\begin{tabular}{ccccc}
\hline$\left[\mathrm{W} / \mathrm{m}^{2}\right]$ & $\begin{array}{c}\text { Summer } \\
\text { Cooling }\end{array}$ & $\begin{array}{c}\text { Winter } \\
\text { Insulating }\end{array}$ & $\begin{array}{c}\text { Summer } \\
\text { Insulating }\end{array}$ & $\begin{array}{c}\text { Winter } \\
\text { Cooling }\end{array}$ \\
\hline $\mathrm{CO}_{2}$ & 117 & 966 & 88 & 883 \\
Air & 15 & 983 & 19 & 655 \\
\hline
\end{tabular}

Nonetheless, if the gas in the cooling skylight would be replaced with air instead of the greenhouse gas, $\mathrm{CO}_{2}$ in this case, is it obvious that the cooling would drop by as much as $85 \%$. The reason for this is that air does not absorb or emit heat radiation and cannot therefore be directly cooled down by the sky. If the winter insulating cases are then compared to each other is the skylight with the $\mathrm{CO}_{2}$ at $\alpha=0.19$ a somewhat better thermal insulator than the skylight containing air. The reason for this is that $\mathrm{CO}_{2}$ is a better conductive insulator than air; however, $\mathrm{CO}_{2}$ is a better transfer medium for convective heat than air and therefore works better in the cooling mode.

So for the skylight to work as attended is it important to choose the thickness of the skylight correctly. The skylight should be thin enough to prevent convective air movement to form when the skylight is in the insulating mode but thick enough that a convective heat flow can occur between the two compartments when the skylight is in its cooling mode. The thickness of $10 \mathrm{~cm}$ has shown to give good results and is therefore used in this study; the width of the skylight is $0.5 \mathrm{~m}$. While then observing Fig.3-a to Fig. 4-b it becomes obvious that the figures presented are not in scale; these distortions allow the figures to be more easily understood without taking too much space. 


\subsection{Skylight filled with $\mathrm{CO}_{2}$}

\subsubsection{Cooling mode}

The goal in the cooling mode is to get the gas inside the skylight to move from the lower compartment to the upper one. This movement should in turn transfer heat from the room to the sky. When observing the simulation results in Fig. 3-a and Fig. 3-b one can observe that this flow is taking place. Additionally, the gas that is moving around in the skylight, as pictured in Fig. 3-a, is clearly cooled down by radiative heat exchange between the sky and the skylight. This is certain as the temperature of this gas flow is lower than that of both the temperature of the room and the temperature of the ambient.

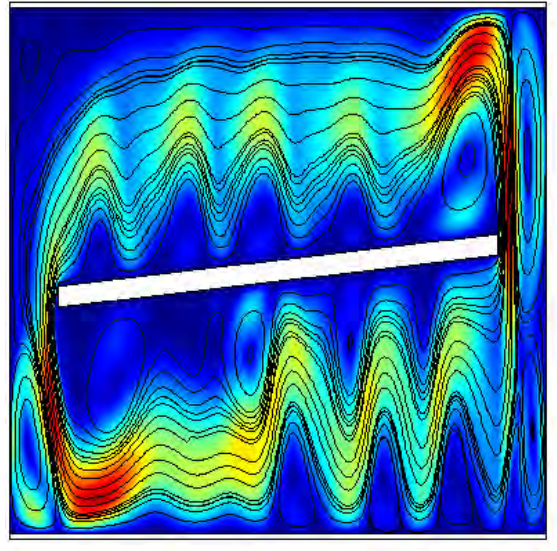

Fig. 3-a. Velocity profile in [cm/s] for cooling mode during summer ${ }^{*}$
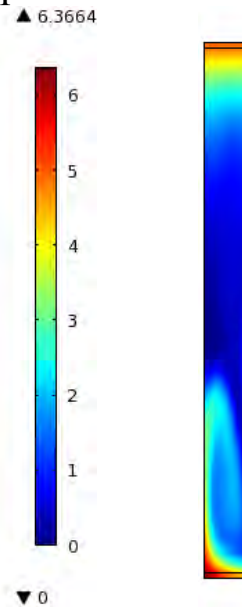

Fig. 3-b. Temperature profile in $\left[{ }^{\circ} \mathrm{C}\right]$ for cooling mode during summer ${ }^{*}$

\subsubsection{Insulating mode}

As mentioned above, for the insulating case to work optimally, the gas movement in the skylight should be kept to a minimum. This, however, is only partially achieved as clear Bérnard cells can be seen in the lower part of the skylight, with also some minor circles in the upper compartment. This suggests that more work could be done to find an optimal depth for the skylight. An interesting phenomenon can be seen in Fig. 4-b where the lowest temperature is achieved in the lower part of the upper compartment. This has to dow ith the high reflectivity of the middle glass window.

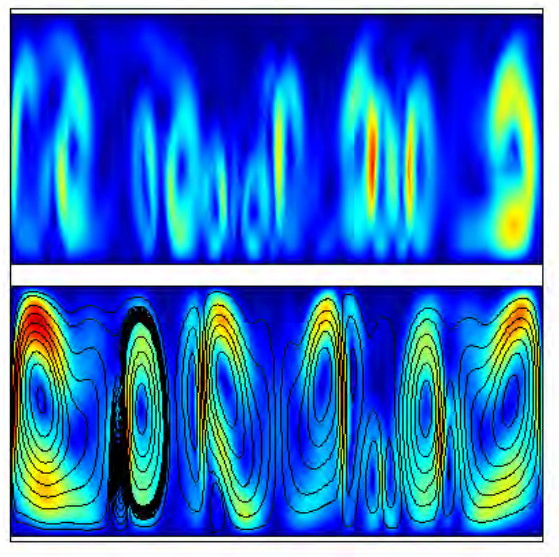

Fig. 4-a. Velocity profile in [cm/s] for insulating mode during winter*

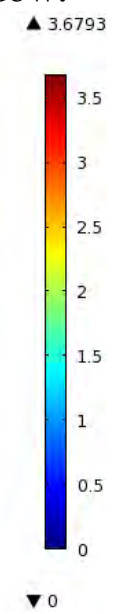

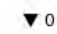

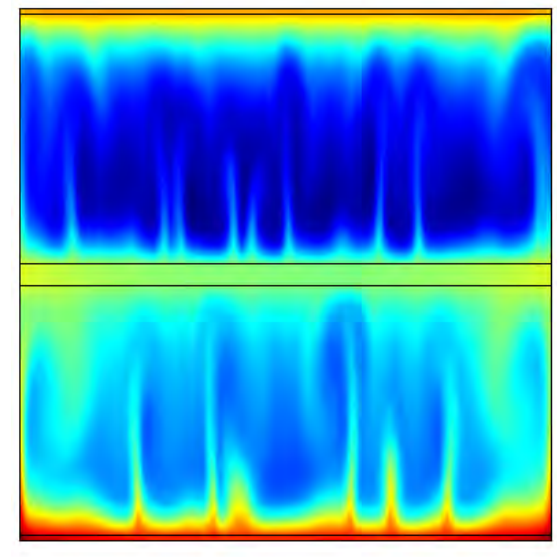

Fig. 4-b. Temperature profile in $\left[{ }^{\circ} \mathrm{C}\right]$ for insulating mode during winter ${ }^{*}$

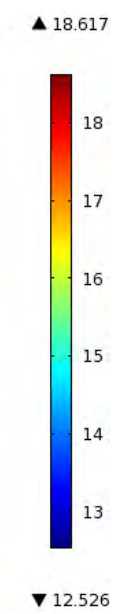

$\mathbf{\nabla} 12.526$

\footnotetext{
${ }^{*}$ Note that the figures are not in scale.
} 


\subsection{Skylight filled with air}

For comparison, the same analyses as presented in chapter 3.1, have also been made for a skylight filled with air.

\subsubsection{Cooling mode}

Even though the skylight is filled with air, a flow pattern is formed between the two compartments; this shown in Fig. 5-a. However, the flow velocities are somewhat slower in Fig. 5-a. than in Fig. 3-a. Furthermore, the temperature profile presented in Fig. 5-b shows that temperatures do not reach lower temperatures than that of the ambient and thus the air is not cooled by the sky.

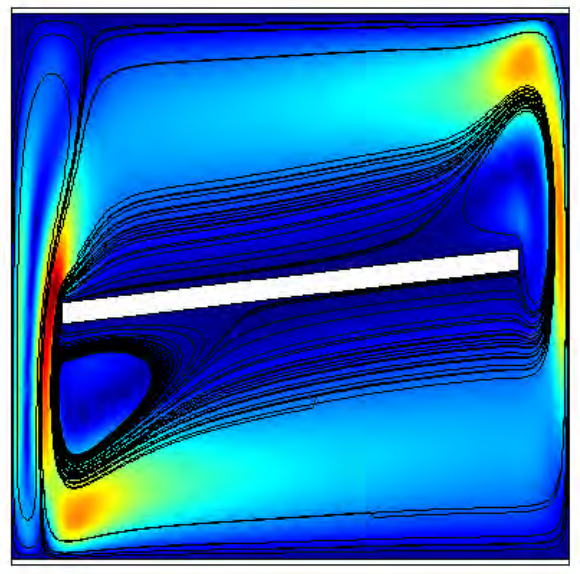

Fig. 5-a. Velocity profile in [cm/s] for cooling mode during summer ${ }^{\dagger}$

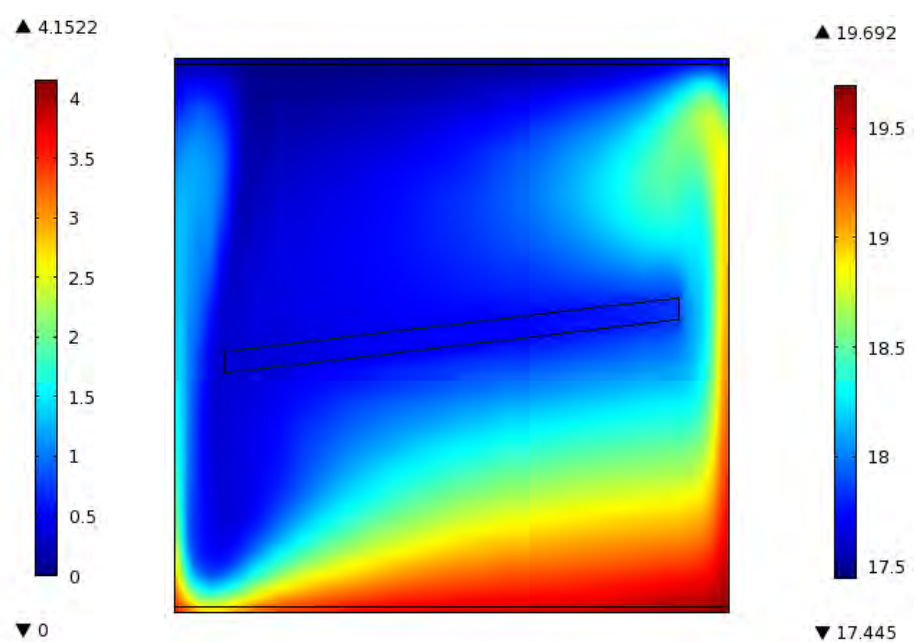

Fig. 5-b. Temperature profile in $\left[{ }^{\circ} \mathrm{C}\right]$ for cooling mode during summer ${ }^{\dagger}$

\subsubsection{Insulating mode}

Interestingly the air filled skylight has the worst insulating properties. The main reason for this is the high air velocities that are presented in Fig. 6-a; another could one could be that the simulation time of 1000 seconds was too short. The temperature profile presented in Fig. 6-a reinforces that temperatures below the ambient are not achievable in a skylight filled with air.

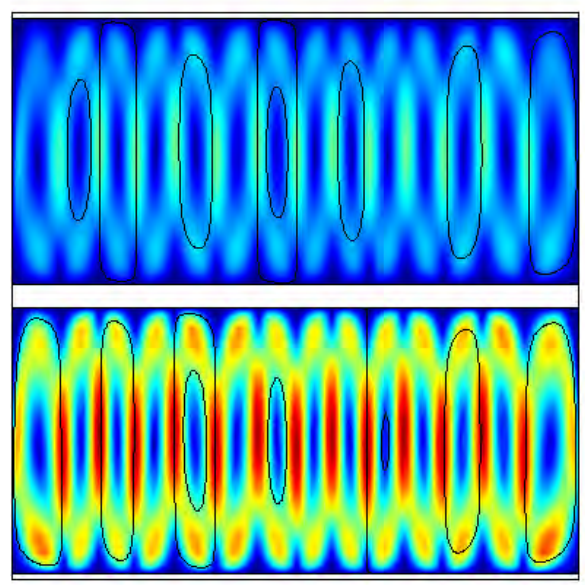

Fig. 6-a. Velocity profile in $[\mathrm{cm} / \mathrm{s}]$ for insulating mode during winter ${ }^{\dagger}$

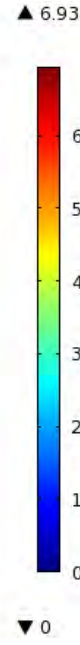

$\nabla 0$

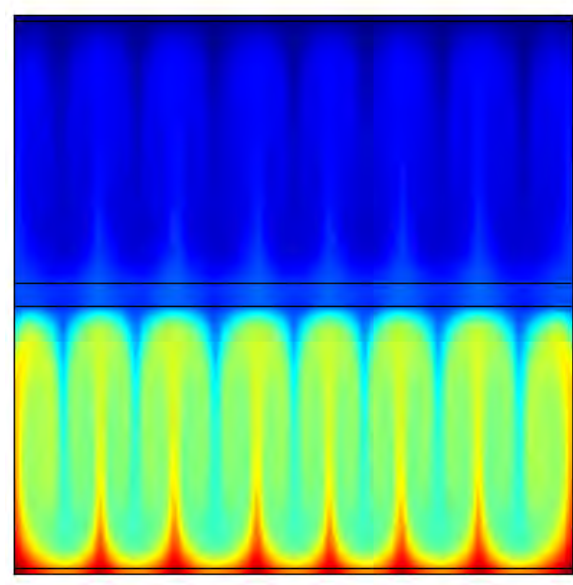

Fig. 6-b. Temperature profile in $\left[{ }^{\circ} \mathrm{C}\right]$ for

insulating mode during winter ${ }^{\dagger}$

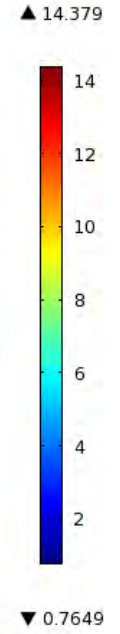

\footnotetext{
${ }^{\dagger}$ Note that the figures are not in scale.
} 


\section{Summary and Conclusions}

This article presents an idea for using a regulated skylight to control heat flow in and out of a space located below. The concept was evaluated using a commercial multiphysics program Comsol.

This study shows that cooling effects of up to $100 \mathrm{~W} / \mathrm{m}^{2}$ are achievable under normal summertime conditions in southern Finland. However, improvements to the design could still be made and thus increase this cooling effect. These improvements would be a result from a careful analysis and optimization of the difference in heat transfer between the two skylight modes. Further improvements to the modeling would be to run Comsol parallel with Matlab so that wavelength dependency could be incorporated into the model, as it is an important factor for radiative heat exchange in participating media. The method chosen for calculating the wavelength dependency would be the exponential wide-band model.

If this system was successfully implemented, savings could be achieved by reducing the use of conventional cooling methods. Unfortunately, window materials that possess the necessary transmission properties typically have poor mechanical properties.

An experimental setup is being designed to assess the modeling results and to study different combinations of materials.

\section{Acknowledgment}

This work is funded by Maj and Tor Nessling Foundation projects 2009301, 2010362 and 2011285 "Solar heat engineering and carbon dioxide: energy recovery using a greenhouse gas", and the Foundation for Åbo Akademi University.

\section{References}

[1] E. Erell, Y. Etzion, Radiative cooling of buildings with flat-plate solar collectors, Build. Environ. 35, 2000, pp. 297 - 305.

[2] M. Rubin, Calculating Heat Transfer Through Windows, Int. J. Energy Res. 6, 1982, pp. $341-349$.

[3] T.S. Eriksson, C.G. Granqvist, J. Karlsson, Transparent thermal insulation with infraredabsorbing gases, Solar Energy Materials. 16, 1987, pp. 243 - 253.

[4] E.M. Lushiku, T.S. Eriksson, A. Hjortsberg, C.G. Granqvist, Radiative cooling to low temperatures with selectively infrared-emitting gases, Solar \& Wind Technology. 1, 1984 , pp. $115-121$.

[5] C.G. Granqvist, Radiative cooling to low temperatures general considerations and applications to selectively emitting SiO films, J. Appl. Phys. 52, 1981, pp. $4205-4220$.

[6] M. Fält, R. Zevenhoven, Radiative cooling in northern Europe using a roof window, Proc. of ES2010, Phoenix, AZ, May, 2010, paper 90192.

[7] G. Rey Colomer, Numerical methods for radiative heat transfer, 2006, pp. 1-161.

[8] R. Siegel, J.R. Howell, Thermal Radiation Heat Transfer, 3rd ed., Hemisphere Pub. Corp., Washington, D.C., 1992 pp. $562-567$. 